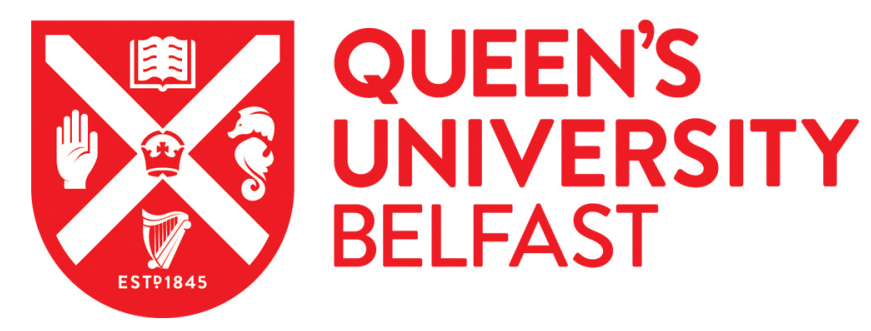

\title{
Atomic oxygen formation in a radio-frequency driven micro- atmospheric pressure plasma jet
}

Waskoenig, J., Niemi, K., Knake, N., Graham, L. M., Reuter, S., Schulz-von Der Gathen, V., \& Gans, T. G. (2010). Atomic oxygen formation in a radio-frequency driven micro-atmospheric pressure plasma jet. Plasma Sources Science and Technology, 19, 045018-045018-11. https://doi.org/10.1088/0963-0252/19/4/045018

Published in:

Plasma Sources Science and Technology

Queen's University Belfast - Research Portal:

Link to publication record in Queen's University Belfast Research Portal

\section{General rights}

Copyright for the publications made accessible via the Queen's University Belfast Research Portal is retained by the author(s) and / or other copyright owners and it is a condition of accessing these publications that users recognise and abide by the legal requirements associated with these rights.

Take down policy

The Research Portal is Queen's institutional repository that provides access to Queen's research output. Every effort has been made to ensure that content in the Research Portal does not infringe any person's rights, or applicable UK laws. If you discover content in the Research Portal that you believe breaches copyright or violates any law, please contact openaccess@qub.ac.uk. 


\title{
Atomic oxygen formation in a radio-frequency driven micro-atmospheric pressure plasma jet
}

\author{
J Waskoenig ${ }^{1}$, K Niemi ${ }^{1}$, N Knake ${ }^{2}$, L M Graham ${ }^{1}$, S Reuter ${ }^{1}$, \\ V Schulz-von der Gathen ${ }^{2}$ and T Gans ${ }^{1}$ \\ ${ }^{1}$ Centre for Plasma Physics, Queen's University Belfast, Belfast BT7 1NN, Northern Ireland, UK \\ ${ }^{2}$ Institute for Experimental Physics II: Applied Plasma Physics, Ruhr-Universität Bochum, \\ 44801 Bochum, Germany \\ E-mail: jwaskoenig01@qub.ac.uk
}

Received 30 January 2010, in final form 16 May 2010

Published 28 June 2010

Online at stacks.iop.org/PSST/19/045018

\begin{abstract}
Atomic oxygen formation in a radio-frequency driven micro-atmospheric pressure plasma jet is investigated using both advanced optical diagnostics and numerical simulations of the dynamic plasma chemistry. Laser spectroscopic measurements of absolute densities of ground state atomic oxygen reveal steep gradients at the interface between the plasma core and the effluent region. Spatial profiles resolving the interelectrode gap within the core plasma indicate that volume processes dominate over surface reactions. Details of the production and destruction processes are investigated in numerical simulations benchmarked by phase-resolved optical emission spectroscopy. The main production mechanisms are electron induced and hence most efficient in the vicinity of the plasma boundary sheath, where electrons are energized. The destruction is driven through chemical heavy particle reactions. The resulting spatial profile of atomic oxygen is relatively flat. The power dependence of the atomic oxygen density obtained by the numerical simulation is in very good agreement with the laser spectroscopic measurements.
\end{abstract}

\section{Introduction}

Cold non-equilibrium atmospheric pressure plasmas have high potential for technological exploitation, primarily due to significant cost reduction and the possibility for treatment of non-vacuum compatible materials [1-6]. Micro-scaled radiofrequency driven atmospheric pressure plasma jets ( $\mu$ APPJs) operated with helium-oxygen feed gas provide high densities of reactive oxygen species and radicals in the order of $10^{21} \mathrm{~m}^{-3}$ at gas temperatures very close to room temperature [7-9]. This is particularly important for treatment of sensitive surfaces in bio-medicine [10-13], e.g. localized skin or wound treatment. Despite enormous potential for technological applications, the fundamentals of these homogeneous non-equilibrium plasmas at ambient pressure are only poorly understood. This is mainly due to the complexity of these plasmas composed of electrodes in the close vicinity of the confining walls and a mixture of neutral and charged atomic and molecular components. Therefore, the understanding of the fundamental plasma chemistry is vital for the development and optimization of such plasma sources. Quantitative measurement of individual particle densities and fluxes provides important net information, but, in most cases, no details about the mechanisms involved.

In this paper, results of absolute atomic oxygen density measurements obtained by two-photon absorption laserinduced fluorescence (TALIF) spectroscopy [14-17] in the regions of the core plasma of the $\mu \mathrm{APPJ}$ are compared with results from a one-dimensional (1D) numerical simulation. The simulation is benchmarked by phase-resolved optical 
emission spectroscopy (PROES) measurements. This comparison yields detailed insight into the plasma chemistry and the formation mechanisms of ground state atomic oxygen.

\section{Experiments}

\section{1. $\mu$ APPJ setup}

The setup of the investigated $\mu$ APPJ consists of two planar stainless steel electrodes of tens of millimetres in length, $1 \mathrm{~mm}$ thickness and $1 \mathrm{~mm}$ distance $[16,18,19]$. One electrode is grounded; the other one is driven at $13.56 \mathrm{MHz}$. The discharge channel of $1 \times 1 \mathrm{~mm}^{2}$ cross section is guided by the electrodes. Both front and back of the entire channel are covered by quartz windows, providing optical access for the applied TALIF spectroscopy. The device is based on the original concept of the APPJ introduced by Selwyn and co-workers [20-22], but scaled down and modified to offer a large solid angle for optical diagnostics. The downscaled dimensions reduce the gas flow $(1 \mathrm{slm})$ significantly, while keeping the gas velocity in the same range of some $10 \mathrm{~ms}^{-1}$. The used gas composition is helium/oxygen (1000:5), unless otherwise noted.

\subsection{TALIF spectroscopy}

The atomic oxygen density is measured by TALIF spectroscopy. Tunable ultra-violet (UV) laser radiation is used to excite oxygen ground state atoms by simultaneous absorption of two UV photons $(\lambda \approx 225 \mathrm{~nm})$. The subsequently emitted fluorescence radiation is measured in order to gain information about the atomic oxygen density. The measurements are calibrated with TALIF measurements on xenon, as introduced in $[14,23]$. Densities of several $10^{19}$ to $10^{20} \mathrm{~m}^{-3}$ can be found, even in the post discharge effluent [16]. Nevertheless, by measuring the densities inside the effluent, it remains unclear whether $\mathrm{O}$ atoms are created only within the actual plasma or also in the effluent, for instance by dissociation of meta-stable molecular states through vacuum UV radiation [15]. It is difficult to estimate the actual densities within the plasma by extrapolation of effluent data due to the interface region between core plasma and effluent. Thus, it becomes mandatory to gain diagnostics access also to the plasma core itself [17].

\subsection{Phase-resolved optical emission spectroscopy}

Information on the electron dynamics within the plasma core of the $\mu$ APPJ can be obtained using PROES [24,25]. Here, temporal and spatial optical emission is measured using a fast gateable ICCD camera with a high repetition rate of several megahertz, allowing information to be collected from each rf-cycle. In this experiment, the emission is spectrally separated using an interference filter with a central wavelength of $840 \mathrm{~nm}$ and a FWHM of $10 \mathrm{~nm}$ to gather information on the emission characteristics of the atomic oxygen optical emission line $\lambda=844 \mathrm{~nm}\left({ }^{3} \mathrm{P} \rightarrow{ }^{3} \mathrm{~S}^{\circ}\right)$. A defined gate width of $2 \mathrm{~ns}$ shifted in $2 \mathrm{~ns}$ steps is used in order to record 37 images during the rf-cycle of $74 \mathrm{~ns}$. The gate time is shifted in $2 \mathrm{~ns}$ increments to sample the entire rf-cycle. Integration times of several million rf-cycles yield a high signal-to-noise ratio.

\section{Numerical simulation}

Since both electrodes of the $\mu$ APPJ have an identical surface area and are capped with dielectric windows along the sides, the discharge produces a symmetric capacitively coupled rf-plasma without dc-selfbias. The electron processes occur symmetric with respect to the centre of the electrode gap, but they are shifted in time by half of the rf-period. In addition, the length of the jet is large compared to the electrode gap, providing plasma chemical equilibrium at typical gas velocities of some $10 \mathrm{~ms}^{-1}$. This offers a model approach that considers only the spatial dimension across the discharge gap. With such a 1D numerical simulation it is possible to get further insight into the electron dynamics and the related atomic oxygen production. The atmospheric pressure plasma is simulated in a helium/oxygen $(1000: 5)$ gas mixture at a gas temperature of $T_{\mathrm{g}}=345 \mathrm{~K}$ [7]. In total, 16 chemical species are considered, namely electrons (e), helium species (meta-stables $\mathrm{He}^{*}$, ions $\mathrm{He}^{+}$, excimers $\mathrm{He}_{2}^{*}$, molecular ions $\mathrm{He}_{2}^{+}$), oxygen species (atoms $\mathrm{O}$, meta-stables $\mathrm{O}\left({ }^{1} \mathrm{D}\right)=\mathrm{O}^{*}$, positive ions $\mathrm{O}^{+}$, negative ions $\mathrm{O}^{-}$, molecular meta-stables $\mathrm{O}_{2}\left({ }^{1} \Delta\right)=\mathrm{O}_{2}^{*}$, positive molecular ions $\mathrm{O}_{2}^{+}$, negative molecular ions $\mathrm{O}_{2}^{-}$and $\mathrm{O}_{3}^{-}$, ozone $\mathrm{O}_{3}$ ) and the background species (helium atoms $\mathrm{He}$, molecular oxygen $\mathrm{O}_{2}$ ). The 116 reactions among those species taken into account are shown in table 1. This set of plasma chemical reactions is compiled from the literature data in [22,26-29]. The selected reactions were found to be the most important for the investigated plasma and parameter regime.

The model is based on fluid equations with a semi-kinetic treatment of the electrons. Here, the transport coefficients and reaction rates for electron-impact collisions are obtained using a zero-dimensional Boltzmann solver in the two-term approximation [30], accounting for a strongly non-Maxwellian electron energy distribution function (EEDF). These rates and transport coefficients are fitted to analytical descriptions as a function of the mean electron energy, which is solved in the $1 \mathrm{D}$ model. The governing equations are mass conservation (1) and momentum conservation (2) for all species, as well as the electron energy conservation (3). The set of coupled differential equations is closed by Poisson's equation:

$$
\begin{aligned}
& \frac{\partial n_{i}}{\partial t}=-\vec{\nabla} \vec{\Gamma}_{i}+\sum_{j} n_{i} n_{j} k_{i j}, \\
& \vec{\Gamma}_{\mathrm{ch}}=\mp n_{\mathrm{ch}} \mu_{\mathrm{ch}} \vec{E}-D_{\mathrm{ch}} \vec{\nabla} n_{\mathrm{ch}} ; \quad \vec{\Gamma}_{\mathrm{n}}=-D_{\mathrm{n}} \vec{\nabla} n_{\mathrm{n}}, \\
& \frac{\partial}{\partial t}\left(n_{\mathrm{e}} \epsilon\right)=-\vec{\nabla} \vec{\Gamma}_{\epsilon}-e \vec{\Gamma}_{\mathrm{e}} \vec{E}-\sum_{j} n_{\mathrm{e}} n_{j} k_{\mathrm{L}, \mathrm{e} j} \\
& \quad-\sum_{x} 3 \frac{m_{\mathrm{e}}}{m_{x}} k_{\mathrm{b}} v_{x} n_{\mathrm{e}}\left(T_{\mathrm{e}}-T_{\mathrm{g}}\right),
\end{aligned}
$$

where

$$
\begin{gathered}
\vec{\Gamma}_{\epsilon}=\frac{5}{3} \vec{\Gamma}_{\mathrm{e}} \epsilon-\frac{5}{3} n_{\mathrm{e}} D_{\mathrm{e}} \vec{\nabla} \epsilon \quad \text { and } \\
x=\mathrm{He}, \mathrm{O}, \mathrm{O}^{*}, \mathrm{O}_{2}, \mathrm{O}_{2}^{*} .
\end{gathered}
$$

Here, $n_{i}$ is the density of the species $i, \vec{\Gamma}_{i}$ the species flux and $k_{i j}$ the rate coefficient. The indices $\mathrm{ch}, \mathrm{n}$, e and $\epsilon$ 
Table 1. Elementary reactions and corresponding rate coefficients.

\begin{tabular}{|c|c|c|c|}
\hline No & Reaction $^{\mathrm{a}}$ & Rate coefficient ${ }^{\mathrm{b}}$ & Reference \\
\hline (R1) & $\mathrm{e}+\mathrm{He} \rightarrow \mathrm{He}+\mathrm{e}$ & $f(\epsilon)$ & [30] \\
\hline (R2) & $\mathrm{e}+\mathrm{He}^{*} \rightarrow \mathrm{He}^{+}+2 \mathrm{e}$ & $f(\epsilon)$ & [31] \\
\hline (R3) & $\mathrm{e}+\mathrm{He} \rightarrow \mathrm{He}^{+}+2 \mathrm{e}$ & $f(\epsilon)$ & [30] \\
\hline (R4) & $\mathrm{e}+\mathrm{He} \rightarrow \mathrm{He}^{*}+\mathrm{e}$ & $f(\epsilon)$ & [30] \\
\hline (R5) & $\mathrm{e}+\mathrm{He}_{2}^{+} \rightarrow \mathrm{He}^{*}+\mathrm{He}$ & $8.9 \times 10^{-15}\left(T_{\mathrm{g}} / T_{\mathrm{e}}\right)^{-1.5}$ & [26] \\
\hline (R6) & $\mathrm{He}_{2}^{*}+\mathrm{M} \rightarrow 2 \mathrm{He}+\mathrm{M}$ & $1.0 \times 10^{4} \mathrm{~s}^{-1}$ & [26] \\
\hline (R7) & $\mathrm{He}_{2}^{*}+\mathrm{He}_{2}^{*} \rightarrow 2 \mathrm{He}+\mathrm{He}_{2}^{+}+\mathrm{e}$ & $1.5 \times 10^{-15}$ & [26] \\
\hline (R8) & $\mathrm{He}^{*}+\mathrm{He}^{*} \rightarrow \mathrm{He}_{2}^{+}+\mathrm{e}^{2}$ & $1.5 \times 10^{-15}$ & {$[26]$} \\
\hline (R9) & $\mathrm{He}^{*}+2 \mathrm{He} \rightarrow \mathrm{He}_{2}^{*}+\mathrm{He}$ & $2.0 \times 10^{-46} \mathrm{~m}^{6} \mathrm{~s}^{-1}$ & [26] \\
\hline (R10) & $\mathrm{He}^{+}+2 \mathrm{He} \rightarrow \mathrm{He}_{2}^{+}+\mathrm{He}$ & $1.1 \times 10^{-43} \mathrm{~m}^{6} \mathrm{~s}^{-1}$ & [26] \\
\hline (R11) & $\mathrm{e}+\mathrm{He}^{+} \rightarrow \mathrm{He}^{*}(+\mathrm{h} v)$ & $5.95 \times 10^{-17} T_{\mathrm{e}}^{-0.5}$ & {$[27]$} \\
\hline (R12) & $2 \mathrm{e}+\mathrm{He}^{+} \rightarrow \mathrm{e}+\mathrm{He}^{*}$ & $1.63 \times 10^{-21} T_{\mathrm{e}}^{-4.5} \mathrm{~m}^{6} \mathrm{~s}^{-1}$ & [27] \\
\hline (R13) & $\mathrm{e}+\mathrm{O}_{2} \rightarrow \mathrm{O}_{2}+\mathrm{e}$ & $f(\epsilon)$ & {$[30]$} \\
\hline (R14) & $\mathrm{e}+\mathrm{O}_{2} \rightarrow \mathrm{O}^{-}+\mathrm{O}$ & $f(\epsilon)$ & [32] \\
\hline (R15) & $\mathrm{e}+\mathrm{O}_{2} \rightarrow 2 \mathrm{O}+\mathrm{e}$ & $f(\epsilon)$ & [33] \\
\hline (R16) & $\mathrm{e}+\mathrm{O}_{2} \rightarrow \mathrm{O}_{2}^{+}+2 \mathrm{e}$ & $f(\epsilon)$ & [30] \\
\hline (R17) & $\mathrm{e}+\mathrm{O}_{2} \rightarrow \mathrm{O}^{+}+\mathrm{O}+2 \mathrm{e}$ & $f(\epsilon)$ & [34] \\
\hline (R18) & $\mathrm{e}+\mathrm{O}_{2}^{+} \rightarrow 2 \mathrm{O}$ & $f(\epsilon)$ & [32] \\
\hline (R19) & $\mathrm{e}+\mathrm{O}_{3} \rightarrow \mathrm{O}^{-}+\mathrm{O}_{2}$ & $f(\epsilon)$ & {$[35]$} \\
\hline (R20) & $\mathrm{e}+\mathrm{O} \rightarrow \mathrm{O}^{+}+2 \mathrm{e}$ & $f(\epsilon)$ & [36] \\
\hline (R21) & $\mathrm{e}+\mathrm{O} \rightarrow \mathrm{O}+\mathrm{e}$ & $f(\epsilon)$ & [36] \\
\hline (R22) & $\mathrm{e}+\mathrm{O}^{-} \rightarrow \mathrm{O}+2 \mathrm{e}$ & $2.2 \times 10^{-20} T_{\mathrm{e}}^{0.5} \exp \left(-26356 / T_{\mathrm{e}}\right)$ & [27] \\
\hline (R23) & $\mathrm{O}^{-}+\mathrm{O}_{2}^{+} \rightarrow \mathrm{O}+\mathrm{O}_{2}$ & $2 \times 10^{-13} 300 / T_{\mathrm{g}}$ & {$[27]$} \\
\hline (R24) & $\mathrm{O}^{-}+\mathrm{O}_{2}^{+} \rightarrow 3 \mathrm{O}$ & $1 \times 10^{-13}$ & [27] \\
\hline (R25) & $\mathrm{O}^{-}+\mathrm{O}^{+} \rightarrow 2 \mathrm{O}$ & $2 \times 10^{-13} 300 / T_{\mathrm{g}}$ & [27] \\
\hline (R26) & $\mathrm{O}^{-}+\mathrm{O}_{2}^{+}+\mathrm{O}_{2} \rightarrow \mathrm{O}+2 \mathrm{O}_{2}$ & $2 \times 10^{-37}\left(300 / T_{\mathrm{g}}\right)^{2.5} \mathrm{~m}^{6} \mathrm{~s}^{-1}$ & [27] \\
\hline (R27) & $\mathrm{O}^{-}+\mathrm{O}^{+}+\mathrm{O}_{2} \rightarrow 2 \mathrm{O}+\mathrm{O}_{2}$ & $2 \times 10^{-37}\left(300 / T_{\mathrm{g}}\right)^{2.5} \mathrm{~m}^{6} \mathrm{~s}^{-1}$ & [27] \\
\hline (R28) & $\mathrm{O}^{-}+\mathrm{O} \rightarrow \mathrm{O}_{2}+\mathrm{e}$ & $2 \times 10^{-16}\left(300 / T_{\mathrm{g}}\right)^{-0.5}$ & {$[27]$} \\
\hline (R29) & $\mathrm{O}^{-}+\mathrm{O}_{2} \rightarrow \mathrm{O}_{3}+\mathrm{e}$ & $5 \times 10^{-18}\left(300 / T_{\mathrm{g}}\right)^{-0.5}$ & [27] \\
\hline (R30) & $\mathrm{O}^{-}+\mathrm{O}_{3} \rightarrow 2 \mathrm{O}_{2}+\mathrm{e}$ & $3.01 \times 10^{-16}\left(300 / T_{\mathrm{g}}\right)^{-0.5}$ & {$[27]$} \\
\hline (R31) & $\mathrm{O}+\mathrm{O}^{+}+\mathrm{O}_{2} \rightarrow \mathrm{O}_{2}^{+}+\mathrm{O}_{2}$ & $1 \times 10^{-42}\left(300 / T_{\mathrm{g}}\right)^{-0.5} \mathrm{~m}^{6} \mathrm{~s}^{-1}$ & [27] \\
\hline (R32) & $\mathrm{O}^{+}+\mathrm{O}_{2} \rightarrow \mathrm{O}_{2}^{+}+\mathrm{O}^{+}$ & $2 \times 10^{-17}\left(300 / T_{\mathrm{g}}\right)^{0.4}$ & [27] \\
\hline (R33) & $\mathrm{O}^{+}+\mathrm{O}_{3} \rightarrow \mathrm{O}_{2}^{+}+\mathrm{O}_{2}$ & $1 \times 10^{-16}$ & [27] \\
\hline (R34) & $2 \mathrm{O}+\mathrm{O}_{2} \rightarrow 2 \mathrm{O}_{2}$ & $2.56 \times 10^{-46}\left(300 / T_{\mathrm{g}}\right)^{0.63} \mathrm{~m}^{6} \mathrm{~s}^{-1}$ & [27] \\
\hline (R35) & $3 \mathrm{O} \rightarrow \mathrm{O}_{2}+\mathrm{O}$ & $9.21 \times 10^{-46}\left(300 / T_{\mathrm{g}}\right)^{0.63} \mathrm{~m}^{6} \mathrm{~s}^{-1}$ & [27] \\
\hline (R36) & $\mathrm{O}+2 \mathrm{O}_{2} \rightarrow \mathrm{O}_{3}+\mathrm{O}_{2}$ & $6 \times 10^{-46}\left(300 / T_{\mathrm{g}}\right)^{2.8} \mathrm{~m}^{6} \mathrm{~s}^{-1}$ & {$[27]$} \\
\hline (R37) & $2 \mathrm{O}+\mathrm{O}_{2} \rightarrow \mathrm{O}_{3}+\mathrm{O}$ & $3.4 \times 10^{-46}\left(300 / T_{\mathrm{g}}\right)^{1.2} \mathrm{~m}^{6} \mathrm{~s}^{-1}$ & {$[27]$} \\
\hline (R38) & $\mathrm{O}_{3}+\mathrm{O} \rightarrow 2 \mathrm{O}+\mathrm{O}_{2}$ & $1.56 \times 10^{-15} \exp \left(-11490 / T_{\mathrm{g}}\right)$ & {$[27]$} \\
\hline (R39) & $\mathrm{O}_{3}+\mathrm{O}_{2} \rightarrow 2 \mathrm{O}_{2}+\mathrm{O}$ & $1.56 \times 10^{-15} \exp \left(-11490 / T_{\mathrm{g}}\right)$ & [27] \\
\hline (R40) & $2 \mathrm{O}_{3} \rightarrow \mathrm{O}_{2}+\mathrm{O}+\mathrm{O}_{3}$ & $1.56 \times 10^{-15} \exp \left(-11490 / T_{\mathrm{g}}\right)$ & {$[27]$} \\
\hline (R41) & $\mathrm{O}_{3}+\mathrm{O}+\mathrm{O}_{2} \rightarrow 2 \mathrm{O}_{3}$ & $2.27 \times 10^{-47} \exp \left(1057 / T_{\mathrm{g}}\right) \mathrm{m}^{6} \mathrm{~s}^{-1}$ & {$[22]$} \\
\hline (R42) & $\mathrm{O}+\mathrm{O}_{3} \rightarrow 2 \mathrm{O}_{2}$ & $1.5 \times 10^{-17} \exp \left(-2250 / T_{\mathrm{g}}\right)$ & {$[22]$} \\
\hline (R43) & $\mathrm{O}^{-}+\mathrm{O}_{2}^{+}+\mathrm{O}_{2} \rightarrow \mathrm{O}_{2}+\mathrm{O}_{3}$ & $2 \times 10^{-37}\left(300 / T_{\mathrm{g}}\right)^{2.5} \mathrm{~m}^{6} \mathrm{~s}^{-1}$ & {$[37]$} \\
\hline (R44) & $\mathrm{He}^{+}+\mathrm{O}^{-} \rightarrow \mathrm{O}+\mathrm{He}$ & $2 \times 10^{-13} 300 / T_{\mathrm{g}}$ & [27] \\
\hline (R45) & $\mathrm{O}^{-}+\mathrm{O}_{2}^{+}+\mathrm{He} \rightarrow \mathrm{O}+\mathrm{O}_{2}+\mathrm{He}$ & $2 \times 10^{-37}\left(300 / T_{\mathrm{g}}\right)^{2.5} \mathrm{~m}^{6} \mathrm{~s}^{-1}$ & [27] \\
\hline (R46) & $\mathrm{O}^{-}+\mathrm{O}^{+}+\mathrm{He} \rightarrow 2 \mathrm{O}+\mathrm{He}$ & $2 \times 10^{-37}\left(300 / T_{\mathrm{g}}\right)^{2.5} \mathrm{~m}^{6} \mathrm{~s}^{-1}$ & {$[27]$} \\
\hline (R47) & $\mathrm{O}^{-}+\mathrm{He}^{+}+\mathrm{He} \rightarrow 2 \mathrm{He}+\mathrm{O}$ & $2 \times 10^{-37}\left(300 / T_{\mathrm{g}}\right)^{2.5} \mathrm{~m}^{6} \mathrm{~s}^{-1}$ & {$[27]$} \\
\hline (R48) & $\mathrm{O}+\mathrm{O}^{+}+\mathrm{He} \rightarrow \mathrm{O}_{2}^{+}+\mathrm{He}$ & $1 \times 10^{-41}\left(300 / T_{\mathrm{g}}\right)^{-0.5} \mathrm{~m}^{6} \mathrm{~s}^{-1}$ & {$[27]$} \\
\hline (R49) & $2 \mathrm{O}+\mathrm{He} \rightarrow \mathrm{O}_{2}+\mathrm{He}$ & $1 \times 10^{-45} \mathrm{~m}^{6} \mathrm{~s}^{-1}$ & {$[27]$} \\
\hline (R50) & $\mathrm{O}+\mathrm{O}_{2}+\mathrm{He} \rightarrow \mathrm{O}_{3}+\mathrm{He}$ & $3.4 \times 10^{-46}\left(300 / T_{\mathrm{g}}\right)^{1.2} \mathrm{~m}^{6} \mathrm{~s}^{-1}$ & {$[27]$} \\
\hline (R51) & $\mathrm{He}^{*}+\mathrm{O}_{2} \rightarrow \mathrm{O}_{2}^{+}+\mathrm{He}+\mathrm{e}$ & $2.54 \times 10^{-16}\left(300 / T_{\mathrm{g}}\right)^{-0.5}$ & [27] \\
\hline (R52) & $\mathrm{He}^{*}+\mathrm{O}_{3} \rightarrow \mathrm{O}_{2}^{+}+\mathrm{O}+\mathrm{He}+\mathrm{e}$ & $2.54 \times 10^{-16}\left(300 / T_{\mathrm{g}}\right)^{-0.5}$ & [27] \\
\hline (R53) & $\mathrm{He}^{*}+\mathrm{O} \rightarrow \mathrm{O}^{+}+\mathrm{He}+\mathrm{e}$ & $2.54 \times 10^{-16}\left(300 / T_{\mathrm{g}}\right)^{-0.5}$ & [27] \\
\hline (R54) & $\mathrm{He}^{+}+\mathrm{O}_{2} \rightarrow \mathrm{O}^{+}+\mathrm{O}+\mathrm{He}$ & $1.07 \times 10^{-15}\left(300 / T_{\mathrm{g}}\right)^{-0.5}$ & [27] \\
\hline (R55) & $\mathrm{He}^{+}+\mathrm{O}_{3} \rightarrow \mathrm{O}^{+}+\mathrm{O}_{2}+\mathrm{He}$ & $1.07 \times 10^{-15}\left(300 / T_{\mathrm{g}}\right)^{-0.5}$ & [27] \\
\hline (R56) & $\mathrm{He}^{+}+\mathrm{O}_{2} \rightarrow \mathrm{O}_{2}^{+}+\mathrm{He}$ & $3.3 \times 10^{-17}\left(300 / T_{\mathrm{g}}\right)^{-0.5}$ & [27] \\
\hline (R57) & $\mathrm{He}^{+}+\mathrm{O} \rightarrow \mathrm{O}^{+}+\mathrm{He}$ & $5 \times 10^{-17}\left(300 / T_{\mathrm{g}}\right)^{-0.5}$ & [27] \\
\hline (R58) & $\mathrm{e}+2 \mathrm{O}_{2} \rightarrow \mathrm{O}_{2}+\mathrm{O}_{2}^{-}$ & $f(\epsilon)$ & [30] \\
\hline (R59) & $\mathrm{e}+\mathrm{O}_{2}+\mathrm{He} \rightarrow \mathrm{He}+\mathrm{O}_{2}^{-}$ & $8.8 \times 10^{-42} T_{\mathrm{e}}^{-0.5} \mathrm{~m}^{6} \mathrm{~s}^{-1}$ & [27] \\
\hline (R60) & $\mathrm{e}+\mathrm{O}_{3} \rightarrow \mathrm{O}+\mathrm{O}_{2}^{-}$ & $f(\epsilon)$ & [35] \\
\hline (R61) & $\mathrm{e}+\mathrm{O}_{3} \rightarrow \mathrm{O}+\mathrm{O}_{2}+\mathrm{e}$ & $f(\epsilon)$ & [28] \\
\hline (R62) & $\mathrm{O}_{2}^{-}+\mathrm{O}_{2}^{+} \rightarrow 2 \mathrm{O}_{2}^{-}$ & $2 \times 10^{-13}\left(300 / T_{\mathrm{g}}\right)$ & [27] \\
\hline (R63) & $\mathrm{O}_{2}^{-}+\mathrm{O}_{2}^{+} \rightarrow \mathrm{O}_{2}+2 \mathrm{O}$ & $1 \times 10^{-13}$ & [27] \\
\hline (R64) & $\mathrm{O}_{2}^{-}+\mathrm{O}^{+} \rightarrow \mathrm{O}_{2}+\mathrm{O}$ & $2 \times 10^{-13}\left(300 / T_{\mathrm{g}}\right)$ & {$[27]$} \\
\hline
\end{tabular}


Table 1. Continued.

\begin{tabular}{|c|c|c|c|}
\hline No & Reaction $^{\mathrm{a}}$ & Rate coefficient ${ }^{\mathrm{b}}$ & Reference \\
\hline (R65) & $\mathrm{O}_{2}^{-}+\mathrm{He}^{+} \rightarrow \mathrm{O}_{2}+\mathrm{He}$ & $2 \times 10^{-13}\left(300 / T_{\mathrm{g}}\right)$ & [27] \\
\hline (R66) & $\mathrm{O}_{3}^{-}+\mathrm{O}_{2}^{+} \rightarrow \mathrm{O}_{3}^{-}+\mathrm{O}_{2}$ & $2 \times 10^{-13}\left(300 / T_{\mathrm{g}}\right)$ & [27] \\
\hline (R67) & $\mathrm{O}_{3}^{-}+\mathrm{O}_{2}^{+} \rightarrow \mathrm{O}_{3}+2 \mathrm{O}$ & $2 \times 10^{-13}$ & [27] \\
\hline (R68) & $\mathrm{O}_{3}^{-}+\mathrm{O}^{+} \rightarrow \mathrm{O}_{3}+\mathrm{O}$ & $2 \times 10^{-13}\left(300 / T_{\mathrm{g}}\right)$ & [27] \\
\hline (R69) & $\mathrm{O}_{3}^{-}+\mathrm{He}^{+} \rightarrow \mathrm{O}_{3}+\mathrm{He}$ & $2 \times 10^{-13}\left(300 / T_{\mathrm{g}}\right)$ & [27] \\
\hline (R70) & $\mathrm{O}^{-}+\mathrm{O}_{3} \rightarrow \mathrm{O}_{3}^{-}+\mathrm{O}$ & $1.99 \times 10^{-16}\left(300 / T_{\mathrm{g}}\right)^{-0.5}$ & [27] \\
\hline (R71) & $\mathrm{O}^{-}+\mathrm{O}_{3} \rightarrow \mathrm{O}_{2}^{-}+\mathrm{O}_{2}$ & $1.02 \times 10^{-17}\left(300 / T_{\mathrm{g}}\right)^{-0.5}$ & [27] \\
\hline (R72) & $\mathrm{O}_{2}^{-}+\mathrm{O} \rightarrow \mathrm{O}_{2}+\mathrm{O}^{-}$ & $1.5 \times 10^{-16}\left(300 / T_{\mathrm{g}}\right)^{-0.5}$ & [27] \\
\hline (R73) & $\mathrm{O}_{2}^{-}+\mathrm{O} \rightarrow \mathrm{O}_{3}+\mathrm{e}$ & $1.5 \times 10^{-16}\left(300 / T_{\mathrm{g}}\right)^{-0.5}$ & [27] \\
\hline (R74) & $\mathrm{O}_{2}^{-}+\mathrm{O}_{3} \rightarrow \mathrm{O}_{2}+\mathrm{O}_{3}^{-}$ & $6 \times 10^{-16}\left(300 / T_{\mathrm{g}}\right)^{-0.5}$ & [27] \\
\hline (R75) & $\mathrm{O}_{3}^{-}+\mathrm{O} \rightarrow \mathrm{O}_{2}^{-}+\mathrm{O}_{2}^{3}$ & $2.5 \times 10^{-16}\left(300 / T_{\mathrm{g}}\right)^{-0.5}$ & [27] \\
\hline (R76) & $\mathrm{e}+\mathrm{He}^{*} \rightarrow \mathrm{He}+\mathrm{e}^{2}$ & $1.099 \times 10^{-17} T_{\mathrm{e}}^{0.31}$ & [29] \\
\hline (R77) & $\mathrm{e}+\mathrm{O}^{*} \rightarrow \mathrm{O}^{*}+\mathrm{e}$ & $f(\epsilon)$ & [38] \\
\hline (R78) & $\mathrm{e}+\mathrm{O}^{*} \rightarrow \mathrm{O}+\mathrm{e}$ & $f(\epsilon)$ & [38] \\
\hline (R79) & $\mathrm{e}+\mathrm{O}^{*} \rightarrow \mathrm{O}^{+}+2 \mathrm{e}$ & $f(\epsilon)$ & {$[38]$} \\
\hline (R80) & $\mathrm{e}+\mathrm{O}_{2} \rightarrow \mathrm{O}^{*}+\mathrm{O}+\mathrm{e}$ & $f(\epsilon)$ & [38] \\
\hline (R81) & $\mathrm{e}+\mathrm{O}_{2} \rightarrow \mathrm{O}_{2}^{*}+\mathrm{e}$ & $f(\epsilon)$ & {$[38]$} \\
\hline (R82) & $\mathrm{e}+\mathrm{O}_{2}^{*} \rightarrow \mathrm{O}_{2}^{*}+\mathrm{e}$ & $f(\epsilon)$ & [38] \\
\hline (R83) & $\mathrm{e}+\mathrm{O}_{2}^{*} \rightarrow \mathrm{O}^{2}+\mathrm{O}^{-}$ & $f(\epsilon)$ & [38] \\
\hline (R84) & $\mathrm{e}+\mathrm{O}_{2}^{*} \rightarrow \mathrm{O}_{2}+\mathrm{e}$ & $f(\epsilon)$ & [38] \\
\hline (R85) & $\mathrm{e}+\mathrm{O}_{2}^{*} \rightarrow 2 \mathrm{O}+\mathrm{e}$ & $f(\epsilon)$ & {$[38]$} \\
\hline (R86) & $\mathrm{e}+\mathrm{O}_{2}^{*} \rightarrow \mathrm{O}^{*}+\mathrm{O}+\mathrm{e}$ & $f(\epsilon)$ & [38] \\
\hline (R87) & $\mathrm{e}+\mathrm{O}_{2}^{*} \rightarrow 2 \mathrm{O}^{*}+\mathrm{e}$ & $f(\epsilon)$ & {$[38]$} \\
\hline (R88) & $\mathrm{e}+\mathrm{O}_{2}^{*} \rightarrow \mathrm{O}_{2}^{+}+2 \mathrm{e}$ & $f(\epsilon)$ & [38] \\
\hline (R89) & $\mathrm{e}+\mathrm{O}_{2}^{*} \rightarrow \mathrm{O}^{2}+\mathrm{O}^{+}+2 \mathrm{e}$ & $f(\epsilon)$ & {$[38]$} \\
\hline (R90) & $\mathrm{e}+\mathrm{O}_{2}^{+} \rightarrow \mathrm{O}^{*}+\mathrm{O}$ & $4.688 \times 10^{-12} T_{\mathrm{e}}^{-0.7}$ & [27] \\
\hline (R91) & $\mathrm{e}+\mathrm{O}^{+} \rightarrow \mathrm{O}^{*}(+\mathrm{h} v)$ & $4.66 \times 10^{-17} T_{\mathrm{e}}^{-0.5}$ & {$[27]$} \\
\hline (R92) & $2 \mathrm{e}+\mathrm{O}^{+} \rightarrow \mathrm{O}^{*}+\mathrm{e}$ & $1.628 \times 10^{-21} T_{\mathrm{e}}^{-4.5} \mathrm{~m}^{6} \mathrm{~s}^{-1}$ & {$[27]$} \\
\hline (R93) & $\mathrm{O}^{-}+\mathrm{O}_{2}^{*} \rightarrow \mathrm{O}_{3}+\mathrm{e}$ & $3 \times 10^{-16}\left(300 / T_{\mathrm{g}}\right)^{0.5}$ & {$[27]$} \\
\hline (R94) & $\mathrm{O}_{2}^{-}+\mathrm{O}_{2}^{*} \rightarrow 2 \mathrm{O}_{2}+\mathrm{e}$ & $2 \times 10^{-16}\left(300 / T_{\mathrm{g}}\right)^{0.5}$ & {$[27]$} \\
\hline (R95) & $\mathrm{O}+\mathrm{O}^{*} \rightarrow 2 \mathrm{O}$ & $8 \times 10^{-18}$ & [27] \\
\hline (R96) & $\mathrm{O}^{*}+\mathrm{O}_{2} \rightarrow \mathrm{O}+\mathrm{O}_{2}^{*}$ & $1.6 \times 10^{-18} \exp \left(-67 / T_{\mathrm{g}}\right)$ & [27] \\
\hline (R97) & $\mathrm{O}^{*}+\mathrm{O}_{2} \rightarrow \mathrm{O}+\mathrm{O}_{2}$ & $4.8 \times 10^{-18} \exp \left(-67 / T_{\mathrm{g}}\right)$ & [27] \\
\hline (R98) & $\mathrm{O}^{*}+\mathrm{O}_{3} \rightarrow \mathrm{O}_{2}+2 \mathrm{O}$ & $1.2 \times 10^{-16}$ & [27] \\
\hline (R99) & $\mathrm{O}^{*}+\mathrm{O}_{3} \rightarrow 2 \mathrm{O}_{2}$ & $1.2 \times 10^{-16}$ & [27] \\
\hline (R100) & $\mathrm{O}^{*}+\mathrm{He} \rightarrow \mathrm{O}+\mathrm{He}$ & $1 \times 10^{-19}$ & [27] \\
\hline (R101) & $\mathrm{O}_{2}^{*}+\mathrm{O}_{2} \rightarrow 2 \mathrm{O}_{2}$ & $3 \times 10^{-24} \exp \left(-200 / T_{\mathrm{g}}\right)$ & [27] \\
\hline (R102) & $2 \mathrm{O}_{2}^{*} \rightarrow 2 \mathrm{O}_{2}$ & $9 \times 10^{-23} \exp \left(-560 / T_{\mathrm{g}}\right)$ & [27] \\
\hline (R103) & $\mathrm{O}_{2}^{*}+\mathrm{O}_{2} \rightarrow \mathrm{O}+\mathrm{O}_{3}$ & $2.95 \times 10^{-27}\left(300 / T_{\mathrm{g}}\right)^{0.5}$ & [27] \\
\hline (R104) & $\mathrm{O}_{2}^{*}+\mathrm{O}_{3} \rightarrow 2 \mathrm{O}_{2}+\mathrm{O}$ & $5.2 \times 10^{-17} \exp \left(-2840 / T_{\mathrm{g}}\right)$ & [27] \\
\hline (R105) & $\mathrm{O}_{2}^{*}+\mathrm{He} \rightarrow \mathrm{O}_{2}+\mathrm{He}$ & $8 \times 10^{-27}\left(300 / T_{\mathrm{g}}\right)^{-0.5}$ & {$[27]$} \\
\hline (R106) & $2 \mathrm{O}+\mathrm{O}_{2} \rightarrow \mathrm{O}_{2}^{*}+\mathrm{O}_{2}$ & $1.93 \times 10^{-47}\left(300 / T_{\mathrm{g}}\right)^{0.63} \mathrm{~m}^{6} \mathrm{~s}^{-1}$ & [27] \\
\hline (R107) & $3 \mathrm{O} \rightarrow \mathrm{O}_{2}^{*}+\mathrm{O}^{2}$ & $6.93 \times 10^{-47}\left(300 / T_{\mathrm{g}}\right)^{0.63} \mathrm{~m}^{6} \mathrm{~s}^{-1}$ & [27] \\
\hline (R108) & $2 \mathrm{O}+\mathrm{He} \rightarrow \mathrm{O}_{2}^{*}+\mathrm{He}$ & $9.88 \times 10^{-47}\left(300 / T_{\mathrm{g}}\right)^{0.63} \mathrm{~m}^{6} \mathrm{~s}^{-1}$ & [27] \\
\hline (R109) & $\mathrm{He}^{*}+\mathrm{O}^{*} \rightarrow \mathrm{O}^{+}+\mathrm{He}+\mathrm{e}$ & $2.54 \times 10^{-16}\left(300 / T_{\mathrm{g}}\right)^{-0.5}$ & {$[27]$} \\
\hline (R110) & $\mathrm{He}^{+}+\mathrm{O}_{2}^{*} \rightarrow \mathrm{O}^{+}+\mathrm{O}+\mathrm{He}$ & $1.07 \times 10^{-15}\left(300 / T_{\mathrm{g}}\right)^{-0.5}$ & {$[27]$} \\
\hline (R111) & $\mathrm{He}^{+}+\mathrm{O}_{2}^{*} \rightarrow \mathrm{O}_{2}^{+}+\mathrm{He}$ & $3.3 \times 10^{-17}\left(300 / T_{\mathrm{g}}\right)^{-0.5}$ & {$[27]$} \\
\hline (R112) & $\mathrm{He}^{+}+\mathrm{O}^{*} \rightarrow \mathrm{O}^{+}+\mathrm{He}$ & $5 \times 10^{-17}\left(300 / T_{\mathrm{g}}\right)^{-0.5}$ & {$[27]$} \\
\hline (R113) & $\mathrm{O}_{2}^{*}+\mathrm{O} \rightarrow \mathrm{O}_{2}+\mathrm{O}$ & $2 \times 10^{-22}$ & {$[27]$} \\
\hline (R114) & $\mathrm{e}+\mathrm{O} \rightarrow \mathrm{O}^{*}+\mathrm{e}$ & $f(\epsilon)$ & [38] \\
\hline (R115) & $\mathrm{O}_{2}^{*}+\mathrm{O}_{3} \rightarrow 2 \mathrm{O}_{2}+\mathrm{O}^{*}$ & $1.01 \times 10^{-17}$ & {$[28]$} \\
\hline (R116) & $\mathrm{O}_{2}^{*}+\mathrm{O}^{-} \rightarrow \mathrm{O}_{2}^{-}+\mathrm{O}$ & $1 \times 10^{-16}$ & [28] \\
\hline
\end{tabular}

${ }^{a} \mathrm{M}$ in reaction (R6) denotes an arbitrary collision partner.

${ }^{\mathrm{b}}$ Units: rate coefficients in $\mathrm{m}^{3} \mathrm{~s}^{-1}$ unless otherwise noted, $T_{\mathrm{e}}$ and $T_{\mathrm{g}}$ in $\mathrm{K} . f(\epsilon)$ indicates that the rate coefficient is calculated on the basis of the two-term approximation EEDF as a function of the mean electron energy [30].

denote charged species, neutral species, electrons and the mean electron energy, respectively. $\mu$ is the mobility, $D$ is the diffusion constant and $\vec{E}$ is the electric field. $k_{\mathrm{L}, \mathrm{e} j}$ in equation (3) represents the loss rate of inelastic collisions, $m_{\mathrm{e}}$ is the electron mass, $m_{x}$ is the mass of the corresponding heavy particle, $k_{\mathrm{b}}$ is the Boltzmann constant, $v_{x}$ is the elastic collision frequency for the corresponding heavy particle, $T_{\mathrm{g}}$ is the gas temperature and $T_{\mathrm{e}}$ is the electron temperature.

The ions are assumed to remain at the same energy (temperature) as the neutral background gas, thus the ion 
energy equation is not solved. This assumption is not fulfilled in the plasma sheath due to the high electric fields present. To account for the higher ion temperature, the generalized Einstein relation is used for ion diffusivity, where the ion temperature is obtained using Wannier's formulation [39]. The momentum conservation is written in the drift diffusion approximation, which is valid at elevated pressure. The main assumptions of this approximation are as follows: the mean velocity of the particles does not change much over time due to the high collision frequency; and the mean free path of the particles is small compared to the Debye length.

The self-consistent model is closed by the boundary conditions for each considered species, potential and electron energy. At the relatively low gas temperature of $T_{\mathrm{g}}=345 \mathrm{~K}$ [7], helium and molecular oxygen are treated as background gases in the simulation with constant densities across the entire gap [29,40,41]. All other neutral species are kinetically limited at the boundaries, i.e. thermal flux onto the electrodes is assumed $\left(\vec{\Gamma}_{\mathrm{n}} \vec{n}=1 / 4 \cdot n_{\mathrm{n}} v_{\mathrm{th}_{\mathrm{n}}}\right.$, here, $v_{\mathrm{th}_{\mathrm{n}}}$ is the thermal velocity of the neutrals and $\vec{n}$ is the normal vector of the boundary). Helium meta-stables and excimers, as well as atomic oxygen meta-stables, are assumed to be lost by deexcitation at the walls (unity surface quenching) [26, 42]. The sticking coefficient for ground state atomic oxygen is in the order of $10^{-4}$ at atmospheric pressure due to surface coverage [43]. The probability of surface recombination of molecular oxygen meta-stables is $10^{-5}$ [27]. Thus, sticking is neglected and zero-surface quenching is assumed for atomic oxygen, molecular oxygen meta-stables and ozone. The positive ions are assumed to be mobility limited at the surface, i.e. the drift term in equation (2) is dominant and the diffusion term is neglected [44]. During each rf-cycle, the sheath collapses only for a short time, therefore the drift of negative ions to the electrodes is neglected, due to their high inertia, and zero density is assumed at the electrodes. For positively charged species, unity surface recombination at the electrodes is assumed. The electron and electron energy flux towards the electrodes are given by the sum of thermal flux and flux due to secondary electron emission from the electrodes. The secondary electron emission coefficient is set to $\gamma=0.1$ and the energy after release from the electrodes to $\epsilon_{\text {wall }}=0.5 \mathrm{eV}$, according to [29]. The boundary condition of Poisson's equation incorporates the fact that one of the electrodes is driven at a frequency of $13.56 \mathrm{MHz}$ and the other is grounded. It is known that mode transitions occur in such capacitively coupled rf-plasmas at atmospheric pressure [46]. In the transition region between the so called $\alpha$ - and $\gamma$-mode, one can obtain multiple solutions for a fixed driving voltage. To be able to cover the transition region and the corresponding multiple solutions, the power is specified rather than the potential of the powered electrode [26,47]. The used transport coefficients for the described boundaries are summarized in table 2. Here, $\mu_{0}$ and $D_{0}$ represent the mobility and diffusion constant at a gas temperature of $T_{\mathrm{g}}=345 \mathrm{~K}$ and atmospheric pressure of $p=1 \times 10^{5} \mathrm{~Pa}$. The described self-consistent model is solved using a time-dependent partial differential equation solver in COMSOL Multiphysics [48] in combination with MATLAB [49] for automatization and post-processing. The
Table 2. Transport coefficients used in the numerical simulation.

\begin{tabular}{llll}
\hline Species & $\mu_{0}\left(\mathrm{~m}^{2} \mathrm{~V}^{-1} \mathrm{~s}^{-1}\right)$ & $D_{0}\left(\mathrm{~m}^{2} \mathrm{~s}^{-1}\right)$ & Reference \\
\hline $\mathrm{e}$ & $f(\epsilon)$ & $f(\epsilon)$ & {$[30]$} \\
$\mathrm{He}^{*}$ & - & $2.02 \times 10^{-4}$ & {$[45]$} \\
$\mathrm{He}^{+}$ & $1.30 \times 10^{-3}$ & Generalized & {$[39]$} \\
& Einstein relation & \\
$\mathrm{He}_{2}^{*}$ & - & $5.86 \times 10^{-5}$ & {$[45]$} \\
$\mathrm{He}_{2}^{+}$ & $2.10 \times 10^{-3}$ & Generalized & {$[39]$} \\
& Einstein relation & \\
$\mathrm{O}$ & - & $1.29 \times 10^{-4}$ & {$[45]$} \\
$\mathrm{O}^{*}$ & - & $1.29 \times 10^{-4}$ & {$[45]$} \\
$\mathrm{O}^{+}$ & $2.85 \times 10^{-3}$ & Generalized & {$[39]$} \\
& & Einstein relation & \\
$\mathrm{O}^{-}$ & $-3.53 \times 10^{-3}$ & Generalized & {$[39]$} \\
& Einstein relation & \\
$\mathrm{O}_{2}^{*}$ & - & $5.51 \times 10^{-5}$ & {$[45]$} \\
$\mathrm{O}_{2}^{+}$ & $2.74 \times 10^{-3}$ & Generalized & {$[39]$} \\
& Einstein relation & \\
$\mathrm{O}_{2}^{-}$ & $-2.71 \times 10^{-3}$ & Generalized & {$[39]$} \\
& Einstein relation & \\
$\mathrm{O}_{3}$ & - & $7.13 \times 10^{-5}$ & {$[45]$} \\
$\mathrm{O}_{3}^{-}$ & $-2.35 \times 10^{-3}$ & Generalized & {$[39]$} \\
& & Einstein relation &
\end{tabular}

Note: $f(\epsilon)$ indicates that the transport coefficients are calculated on the basis of the two-term approximation EEDF as a function of the mean electron energy [30].

timescales of the electron dynamics (several nanoseconds) and the plasma chemistry (several milliseconds) are substantially different. Therefore, both are decoupled: first the complete set of partial differential equations is solved for several rf-cycles, and then only the neutral species reactions are solved on a much longer timescale. This is done iteratively until convergence is reached.

\section{Results}

\subsection{Benchmark of the numerical simulation by PROES}

Figure 1 shows the spatio-temporal emission characteristics of the atomic oxygen optical emission line $\lambda=844 \mathrm{~nm}$ $\left({ }^{3} \mathrm{P} \rightarrow{ }^{3} \mathrm{~S}^{\mathrm{o}}\right)$ across the electrode gap during one rf-cycle. Subfigure $(a)$ is obtained by the self-consistent model and (b) by PROES measurements. Both are determined at powers close to the onset of the $\gamma$-mode. The simulated emission in figure $1(a)$ is calculated using the sum of the direct and dissociative electron-impact excitation coefficients of the $\mathrm{O}\left({ }^{3} \mathrm{P}\right)$ state with the time and space dependent electron density, and integrating the resulting time dependence to account for the effective lifetime of the upper state [50]. At atmospheric pressure conditions it is crucial to account for collisional de-excitation (quenching) of the investigated excited state in addition to the spontaneous emission [51]. The effective lifetime of the upper state is calculated to be $\tau_{\text {eff }}=\left(\tau^{-1}+\sum_{q} n_{q} k_{q}\right)^{-1}=5.43 \mathrm{~ns}$, which is significantly shorter than the natural lifetime of $\tau=34.7 \mathrm{~ns}$. Here, $n_{q}$ and $k_{q}$ are the density of the quenching partner (oxygen, helium) and the corresponding quenching rate coefficient taken from [14]. Cascade processes populating the $\mathrm{O}\left({ }^{3} \mathrm{P}\right)$ state are neglected, since higher lying states are in general more 

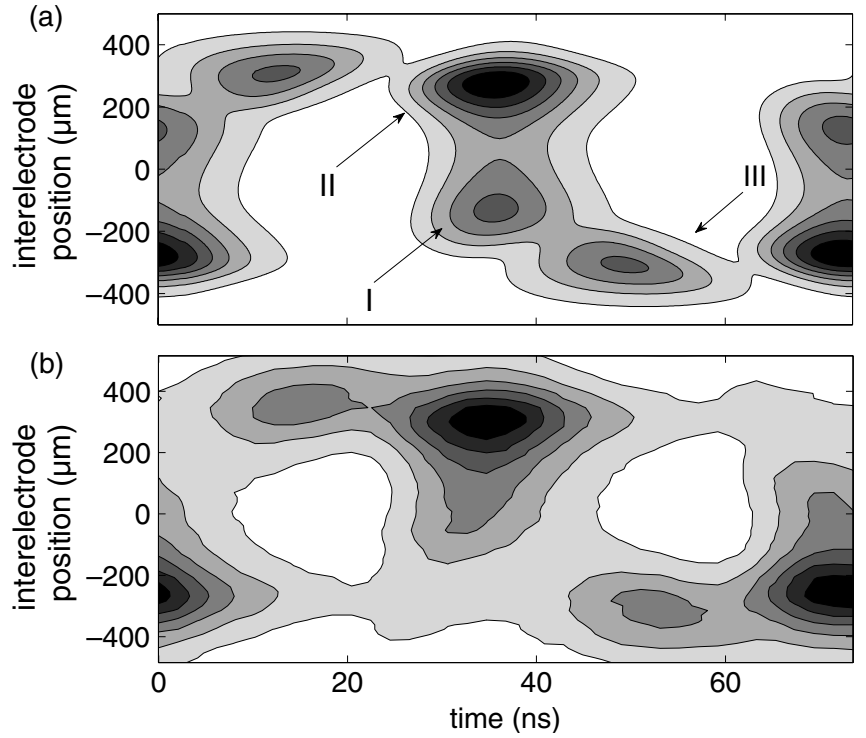

Figure 1. $\mathrm{O}\left({ }^{3} \mathrm{P} \rightarrow{ }^{3} \mathrm{~S}^{\mathrm{o}}\right)$ line emission pattern in helium/oxygen $(1000: 5)$ within one $13.56 \mathrm{MHz}$ cycle and the electrode gap on a linear grey scale starting from zero. Results of $(a)$ the numerical simulation and $(b)$ the PROES measurement.

efficiently quenched by collisions, due to their longer lifetime, particularly at atmospheric pressure. Here, three different emission patterns can be observed. The dominant emission structures can be associated with the sheath expansion (I) and sheath collapse (II). During sheath expansion, electrons are accelerated away from the electrode by the fast moving sheath edge, whilst at the opposite electrode, the intensive sheath collapse structure is induced by a field reversal effect. This reversal is due to electrons colliding with the background gas, thus hindering them from responding to the rapidly changing sheath potential. Therefore, a space charge builds up, which leads to an electric field accelerating the electrons towards the electrodes. In addition a third maximum is observed within the plasma boundary sheath, close to the electrodes (III). This is due to secondary electron multiplication as a result of the high electric fields in this region. These secondary electrons are generated by heavy particle impact at the electrodes as well as by Penning ionization in the plasma volume (reactions: (R7), (R8), (R51), (R52), (R53) and (R109)).

Figure $1(b)$ shows the experimentally determined spatiotemporal characteristics of the $844 \mathrm{~nm}$ emission line at the same operating parameters as used for the numerical simulation. In the case of the atomic oxygen optical emission line at $\lambda=844 \mathrm{~nm}$, the short effective lifetime of $\tau_{\text {eff }}=5.43 \mathrm{~ns}$ allows the resolution of the fast electron dynamics within the rf-cycle $\left(T_{\mathrm{rf}}=73.74 \mathrm{~ns}\right)$. In each half cycle the three emission structures correspond well in shape and position to the results of the numerical simulation.

Compared with similar simulations and experiments in low pressure capacitively coupled rf-plasmas [24, 52], the main difference is the relative strong emission in the discharge centre. This emission cannot be explained by the sheath expansion as a single excitation mechanism. The accelerated electrons lose their energy within a few micrometres in this highly collisional regime. Therefore, one would expect
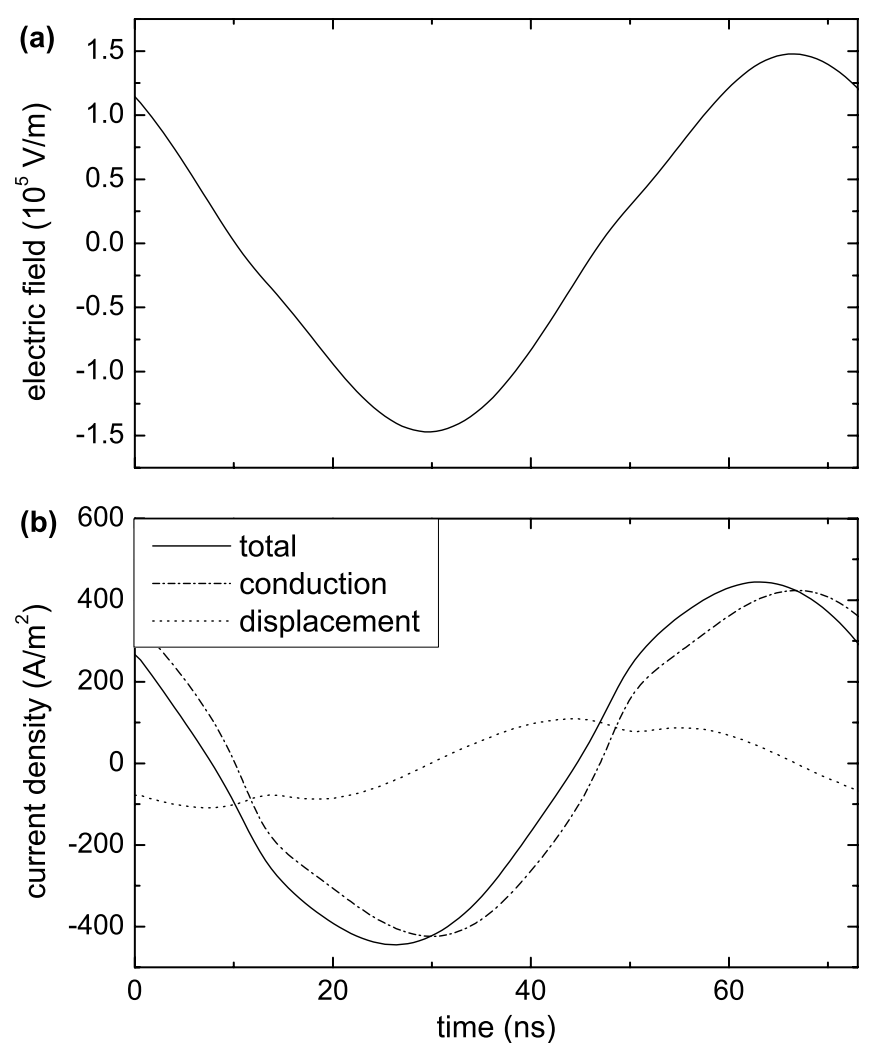

Figure 2. (a) Evolution of the simulated electric field as well as (b) the simulated total, conduction and displacement current density in the discharge centre over one rf-cycle.

confined emission structures near the sheath edge. The numerical simulations reveal that the additional emission in the discharge centre can be explained by a comparatively high electric field present in the plasma bulk region at atmospheric pressure conditions. The bulk electric field oscillates between $\pm 1.5 \times 10^{5} \mathrm{~V} \mathrm{~m}^{-1}$ (figure $2(a)$ ). These high field values are comparable to sheath electric fields of low pressure plasmas [53]. The electric field drives electrons produced during sheath expansion through the discharge gap. Thus, effective electron production in the plasma bulk, even at elevated pressure, is possible. Under these conditions the displacement current density in the discharge centre is in the order of the conduction current density (figure 2(b)). Thus, the total current is shifted in phase. In contrast to its low pressure counterparts, the displacement current in the plasma bulk is not negligible for atmospheric pressure plasmas.

\subsection{Spatial $O$ density profiles}

Figure 3 shows the measured atomic oxygen density along the centre of the plasma channel of the $\mu \mathrm{APPJ}$ at $12 \mathrm{~W}$ generator power and a gas flow of $1.5 \mathrm{slm} \mathrm{He}$ and $9 \mathrm{sccm}$ $\mathrm{O}_{2}$, thus a helium/oxygen ratio of $1000: 6$. Here, the gas channel of the $\mu$ APPJ is guided by the electrodes up to $z=40 \mathrm{~mm}$ and onwards continued by BK7 glass substrates. This allows an undisturbed measurement along the gas channel from the plasma core into the effluent of the jet. The density remains constant at $2.7 \times 10^{21} \mathrm{~m}^{-3}$ up to the electrode edge at $z=40 \mathrm{~mm}$, then it starts decreasing exponentially towards 


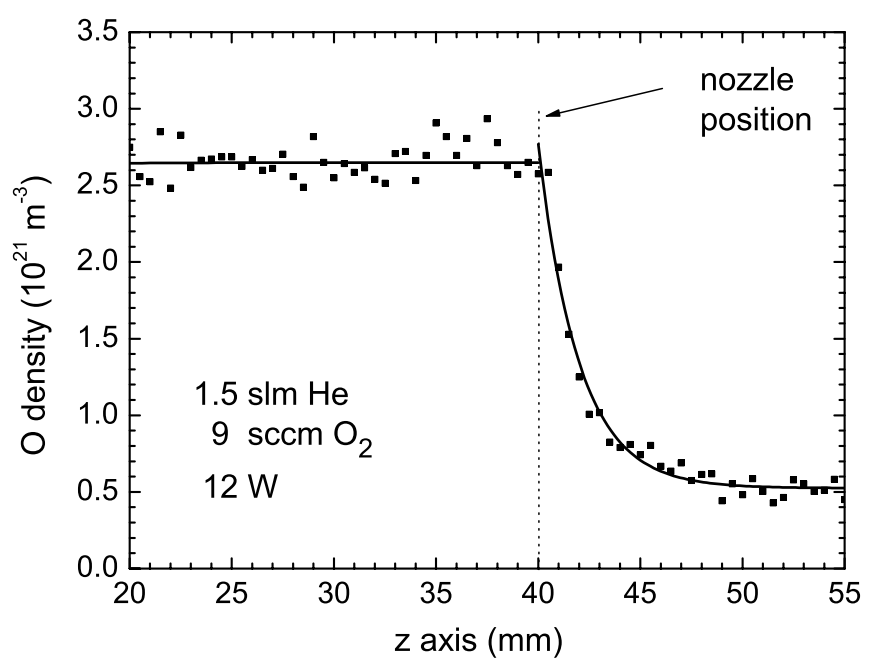

Figure 3. Axial profile of the measured atomic oxygen density at a gas composition of helium/oxygen $(1000: 6)$ from the discharge centre at $z=20 \mathrm{~mm}$ into the effluent starting at $z=40 \mathrm{~mm}$. Decay shows an exponential behaviour.

a constant value of $\approx 5 \times 10^{20} \mathrm{~m}^{-3}$. Thus, a substantial $\mathrm{O}$ concentration is transported far outside into the effluent region. The amount of transported atomic oxygen depends on the atomic oxygen density generated within the plasma core. Therefore, it is crucial to get detailed information about the $\mathrm{O}$ formation mechanisms inside the $\mu$ APPJ.

Figure 4(a) shows a simulated profile of the atomic oxygen density across the interelectrode gap. The obtained profile is relatively flat due to the assumption of zero-surface quenching of $\mathrm{O}$ at the electrode surfaces at $x=-500 \mu \mathrm{m}$ and $x=$ $500 \mu \mathrm{m}$. Figure $4(b)$ shows a comparison of the simulated profile with profiles obtained by TALIF measurements (same conditions as in figure 3) across the interelectrode gap. The TALIF signal of atomic oxygen (triangles) was recorded during plasma operation. For the reference measurement (open circles), the device was simply flooded with xenon gas to achieve a homogeneous density distribution [14]. It can be seen that the signal of the reference gas decreases towards the electrodes. This decrease is due to geometric vignetting of the solid angle of detection by the electrodes during the TALIF measurement. To compare the atomic oxygen TALIF measurements with the numerical simulation results, the atomic oxygen profile obtained from the simulation is multiplied by the relative TALIF signal of the xenon reference gas (squares). It can be seen that the relative profiles agree quite well within the given signal-to-noise ratio. The assumption of zero-surface quenching for atomic oxygen at the electrodes is validated by this agreement between simulation and TALIF measurement. Thus, volume processes (particleparticle reactions) dominate over plasma boundary induced processes (particle-wall interaction) in the atomic oxygen production and destruction within the core plasma of the $\mu$ APPJ.

\subsection{Power dependency of the $O$ density}

Figure 5 compares the atomic oxygen density dependency on the power obtained from the numerical simulation and
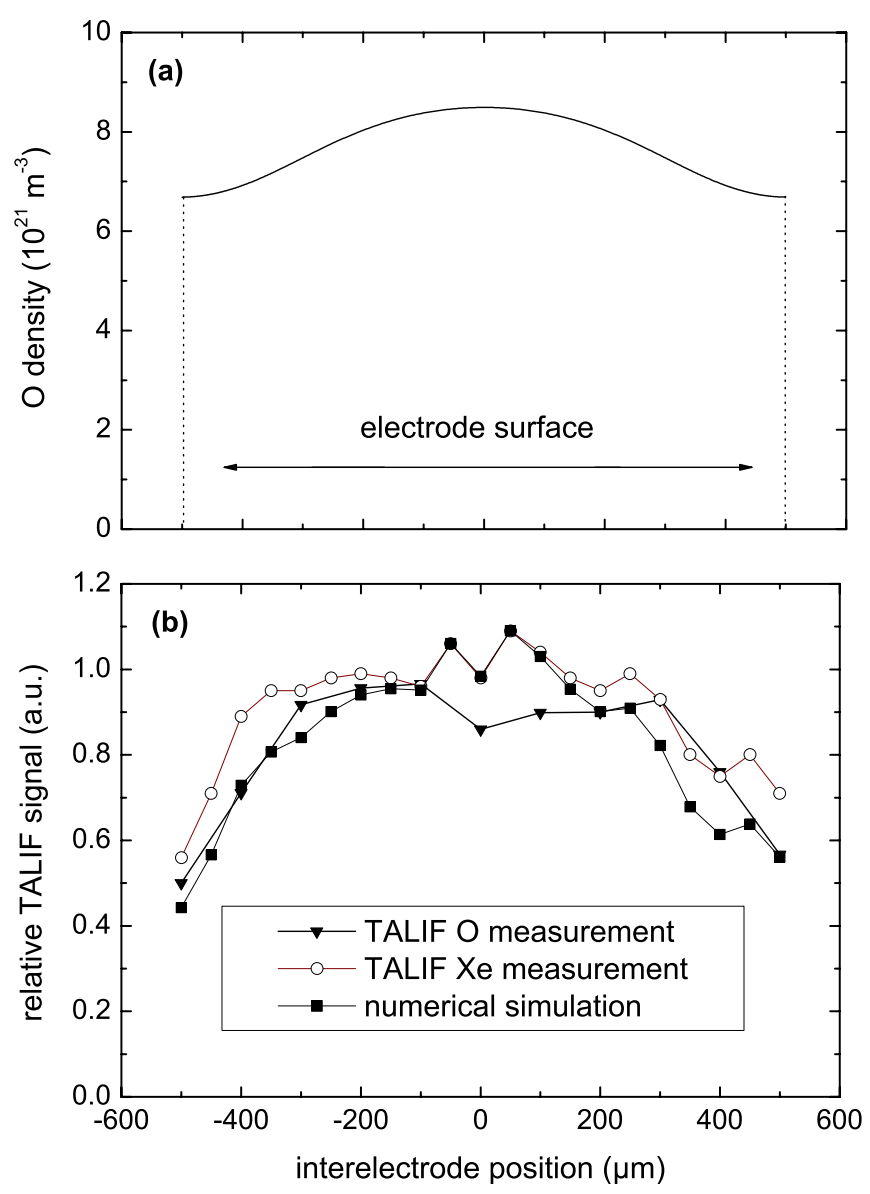

Figure 4. (a) Time averaged simulated ground state atomic oxygen density as well as $(b)$ relative TALIF signals of atomic oxygen and xenon.

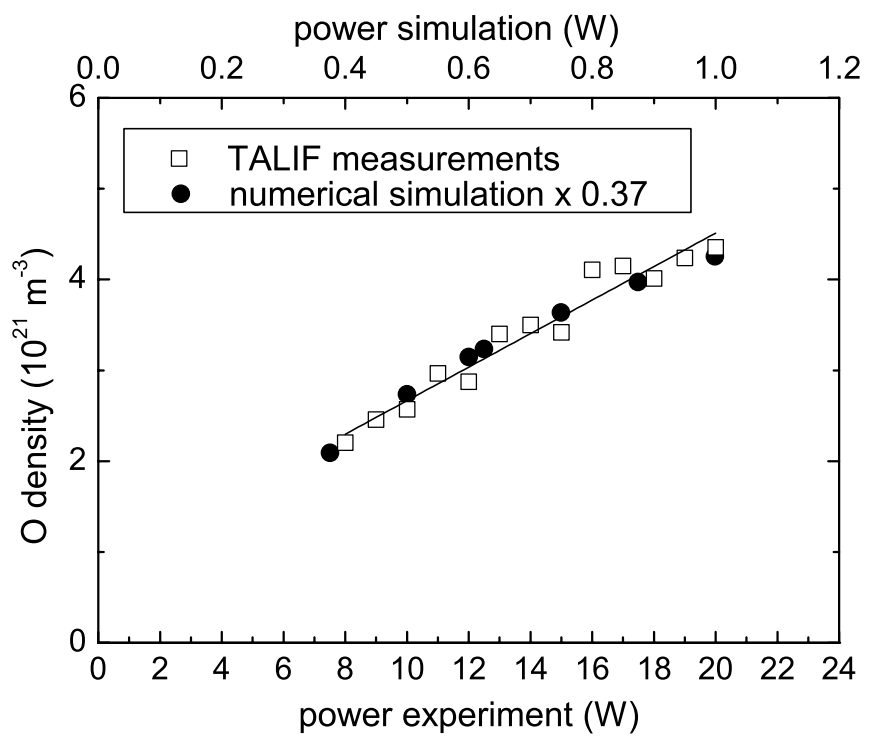

Figure 5. O density in the discharge centre under power variation.

the TALIF spectroscopic measurements. The two separate power axes differ significantly from each other. First, only a small fraction of the generator power is actually coupled into the plasma, since there are losses in the connecting cables, matching network and heating of the electrodes, as well as by rf-radiation of the jet device acting as an antenna 
[54]. Secondly, the power set in the numerical simulation underestimates the actual power input, since the 1D description neglects losses of the considered species by recombination at the quartz-glass panes of the jet and by convection due to the gas flux perpendicular to the simulation domain. Both axes are matched with the onset of the $\gamma$-mode. In the experiment, this point is determined by the transition into a constricted high density plasma. In the simulation, the onset can be identified when the slope of the voltage-power characteristics changes from positive to negative [41].

The values obtained from the numerical simulation are scaled down by a factor 0.37 to illustrate the very good qualitative agreement of the measured and the simulated dependency. The factor between those values can be explained, taking the uncertainties of the two different approaches for the determination into account. The overall reliability of the TALIF measurements is stated to be within 50\% [14]. The result of the numerical simulation, on the other hand, depends on a large variety of reaction constants for heavy particle interactions and cross-sections for electron-impact reactions, the latter having an uncertainty of at least 30\% [51]. The numerical simulation results show that the atomic oxygen density increases slightly sub-linear with power. This cannot be resolved in the TALIF measurements due to the limited signal-to-noise ratio.

The comparison with PROES and TALIF results shows that the employed numerical simulation describes the qualitative behaviour of the electron dynamics and the plasma chemistry within the core of the $\mu$ APPJ very well. Using the benchmarked simulation, it is now possible to get further insight into the details of the formation mechanisms of atomic oxygen.

\subsection{Formation mechanisms of $O$}

Figure 6 shows the most important (a) destruction and (b) production mechanisms of atomic oxygen time averaged over one rf-cycle across the discharge gap at an input power of $0.6 \mathrm{~W}$, which corresponds to a generator power of $12 \mathrm{~W}$ in the experiment (comparable to figures 3 and 4). The main destruction mechanisms shown in figure $6(a)$ are all heavy particle driven. These are in descending order of relevance:

$$
\begin{gathered}
\mathrm{O}+\mathrm{O}_{2}+\mathrm{He} \rightarrow \mathrm{O}_{3}+\mathrm{He}, \\
2 \mathrm{O}+\mathrm{He} \rightarrow \mathrm{O}_{2}+\mathrm{He} \\
\mathrm{O}+\mathrm{O}_{3} \rightarrow 2 \mathrm{O}_{2}
\end{gathered}
$$

The reaction rates of (R49) and (R50) are similar to the spatial profile of the atomic oxygen density shown in figure $4(b)$. For (R50) it is proportional, whereas the rate of (R49) goes with the square of the $\mathrm{O}$ density profile. In case of the ozone induced recombination (R42), the atomic oxygen profile is multiplied by the ozone profile. The latter decreases from the electrodes towards the discharge centre, leading to the overall flat profile of the reaction rate of (R42).

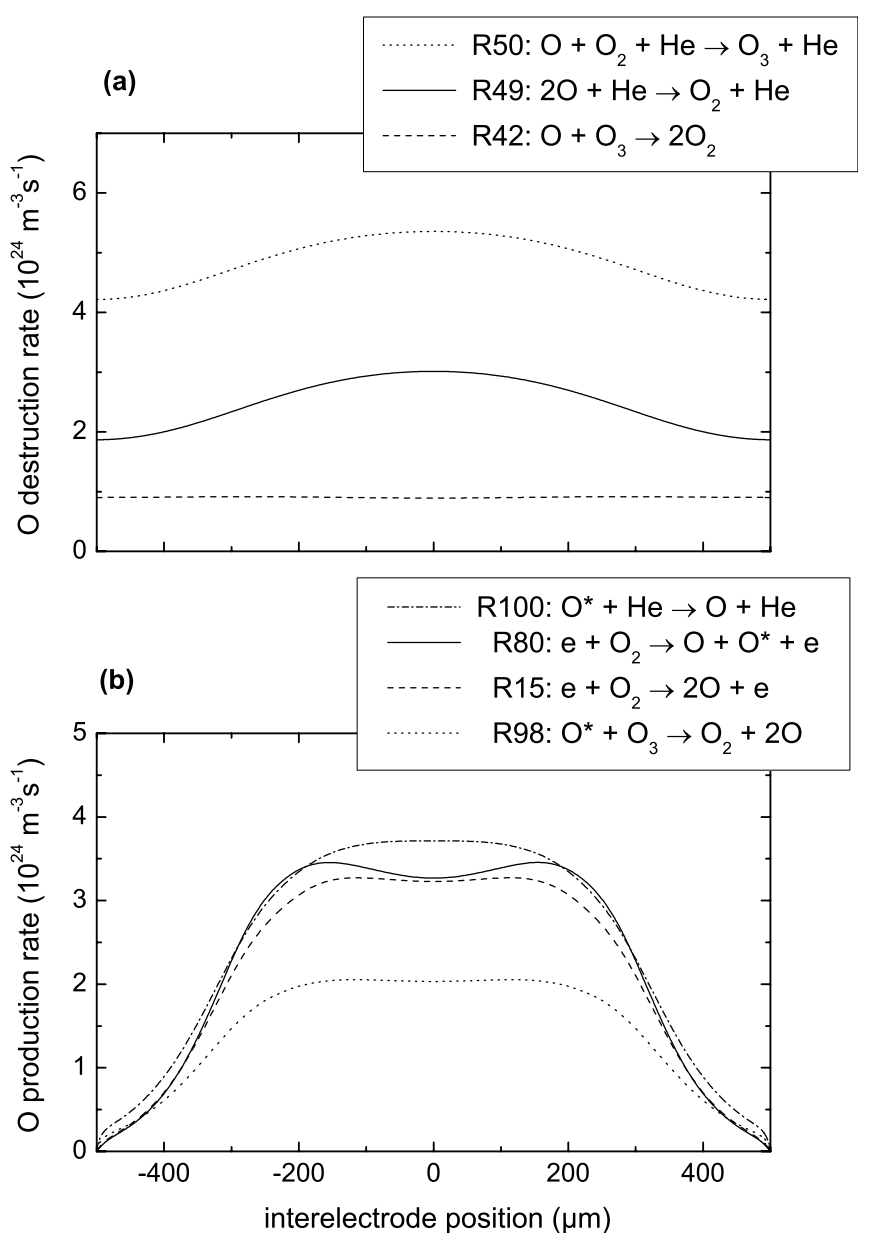

Figure 6. Most important $(a)$ destruction and $(b)$ production mechanisms of atomic oxygen time averaged over one rf-cycle across the discharge gap at an input power of $0.6 \mathrm{~W}$.

The production, on the other hand, is driven by electron and heavy particle induced processes:

$$
\begin{gathered}
\mathrm{O}^{*}+\mathrm{He} \rightarrow \mathrm{O}+\mathrm{He} \\
\mathrm{e}+\mathrm{O}_{2} \rightarrow \mathrm{O}+\mathrm{O}^{*}+\mathrm{e} \\
\mathrm{e}+\mathrm{O}_{2} \rightarrow 2 \mathrm{O}+\mathrm{e} \\
\mathrm{O}^{*}+\mathrm{O}_{3} \rightarrow \mathrm{O}_{2}+2 \mathrm{O}
\end{gathered}
$$

Reaction (R100) has the same profile as the atomic oxygen meta-stable density, since helium is treated as a uniformly distributed background gas. The ozone dissociation (R98) has a very small dip in the centre, caused by the ozone density comparable to reaction (R42). The dissociative excitation of atomic oxygen (R80) shows a double peak structure, as can be seen in figure $6(b)$. These two peaks are induced by the sheath motion represented by the electron dynamics from the PROES measurements (figure 1). The dissociation (R15) has only a very slight double peak structure due to a different energy threshold. The dissociation has a lower threshold than the dissociative excitation, i.e. more low 
energetic electrons from the plasma bulk region can contribute to the dissociation, resulting in the time averaged profile in figure 6(b). Electron and heavy particle driven processes each contribute approximately $50 \%$ to the total production of atomic oxygen. In figure $7(a)$ the electron and heavy particle induced production rates of atomic oxygen are compared to the total production rate for several input powers. It can be seen that the relative contributions are comparable for all powers and that the electron induced production rates are a bit higher than the heavy particle induced processes. The latter ((R98) and (R100)) are both dependent on the atomic oxygen meta-stable density. The main destruction processes of atomic oxygen meta-stables in descending order of relevance are

$$
\begin{gathered}
\mathrm{O}^{*}+\mathrm{He} \rightarrow \mathrm{O}+\mathrm{He}, \\
\mathrm{O}^{*}+\mathrm{O}_{3} \rightarrow 2 \mathrm{O}_{2}, \\
\mathrm{O}^{*}+\mathrm{O}_{3} \rightarrow 2 \mathrm{O}+\mathrm{O}_{2}, \\
\mathrm{O}^{*}+\mathrm{O}_{2} \rightarrow \mathrm{O}+\mathrm{O}_{2}, \\
\mathrm{O}^{*}+\mathrm{O}_{2} \rightarrow \mathrm{O}+\mathrm{O}_{2}^{*}, \\
\mathrm{O}^{*}+\mathrm{O} \rightarrow 2 \mathrm{O},
\end{gathered}
$$

Integration over the 1D simulation domain of the total volumetric losses allows comparison with the loss of atomic oxygen meta-stables by thermal flux to the electrodes. The surface quenching is found to be below $0.1 \%$ of the integrated volumetric losses, hence it can be neglected. The production mechanisms are electron and heavy particle driven, as in the case of atomic oxygen:

$$
\begin{gathered}
\mathrm{e}+\mathrm{O}_{2} \rightarrow \mathrm{O}+\mathrm{O}^{*}+\mathrm{e} \\
\mathrm{O}_{2}^{*}+\mathrm{O}_{3} \rightarrow 2 \mathrm{O}_{2}+\mathrm{O}^{*} \\
\mathrm{e}+\mathrm{O} \rightarrow \mathrm{O}^{*}+\mathrm{e}
\end{gathered}
$$

The contributions of the electron and heavy particle induced production mechanisms of $\mathrm{O}^{*}$ compared to the total production are shown in figure $7(b)$. Both contribute approximately equally over the entire power range, whereas the electron induced processes are slightly higher. The heavy particle reaction (R115) is dependent on the $\mathrm{O}_{2}^{*}$ density. The destruction of $\mathrm{O}_{2}^{*}$ is mainly driven by reaction ( $\left.\mathrm{R} 115\right)$. The surface losses in this case are lower than $10 \times 10^{-6}$ compared to the integrated sum of volumetric losses. The main production processes in descending order of importance are

$$
\begin{gathered}
\mathrm{e}+\mathrm{O}_{2} \rightarrow \mathrm{O}_{2}^{*}+\mathrm{e}, \\
\mathrm{O}^{*}+\mathrm{O}_{2} \rightarrow \mathrm{O}_{2}^{*}+\mathrm{O}, \\
2 \mathrm{O}+\mathrm{He} \rightarrow \mathrm{O}_{2}^{*}+\mathrm{He} .
\end{gathered}
$$
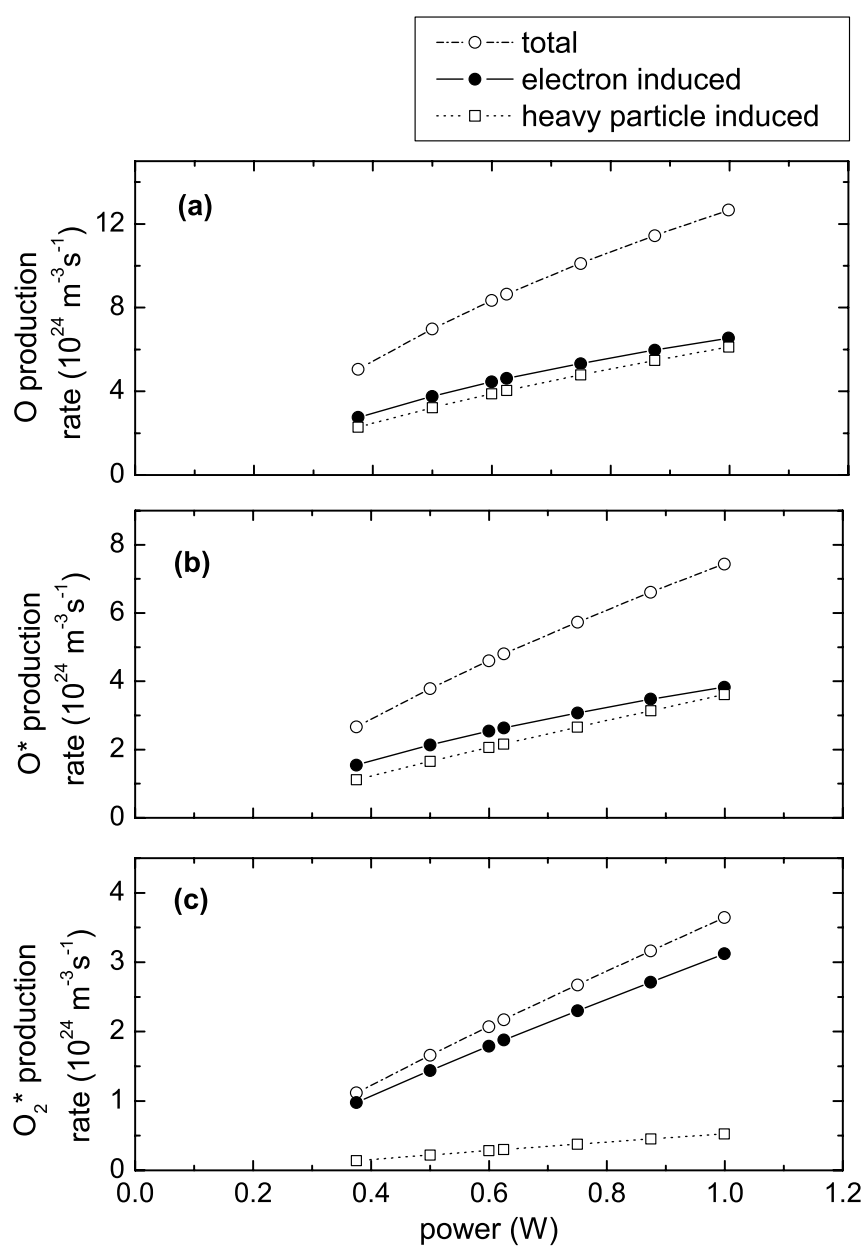

Figure 7. Time and space averaged total, electron and heavy particle induced production mechanisms of $(a)$ atomic oxygen, $(b)$ atomic oxygen meta-stables and $(c)$ molecular oxygen meta-stables.

The electron-impact excitation (R81) is the dominant production process. Figure $7(c)$ shows that $15 \%$ of total $\mathrm{O}_{2}^{*}$ production is related to the heavy particle reactions ((R96) and (R108)) and $85 \%$ to the electron driven production.

The production of $\mathrm{O}^{*}$ is $50 \%$ driven by the heavy particle reaction ( $\mathrm{R} 115)$ and $50 \%$ by electron-impact reactions. $\mathrm{O}_{2}^{*}$ is almost exclusively produced by the electron-impact excitation (R81). Hence, the production of $\mathrm{O}^{*}$ through reaction (R115) is indirectly driven by electrons. Here, $\mathrm{O}_{2}^{*}$ serves as a precursor for the $\mathrm{O}^{*}$ production. The total production of $\mathrm{O}^{*}$ is directly $((\mathrm{R} 80)$ and (R114)) or indirectly (R115) driven by electrons. The heavy particle induced production of $\mathrm{O}$ is further highly dependent on the $\mathrm{O}^{*}$ density. Therefore, the production of $\mathrm{O}$ through reactions (R100) and (R98) is also indirectly driven by electrons. In this case, $\mathrm{O}^{*}$ serves as a precursor for $\mathrm{O}$ production. Hence, the formation of atomic oxygen is, as expected, given by electron driven production, either direct or indirect using $\mathrm{O}^{*}$ and $\mathrm{O}_{2}^{*}$ as precursors, and heavy particle driven destruction. Taking into account only the most important production and destruction channels for $\mathrm{O}((\mathrm{R} 100)$, (R80), (R15) and (R50)), O* ((R80), (R115) and (R100)) and $\mathrm{O}_{2}^{*}((\mathrm{R} 81)$ and (R115)), one can derive the following expression 


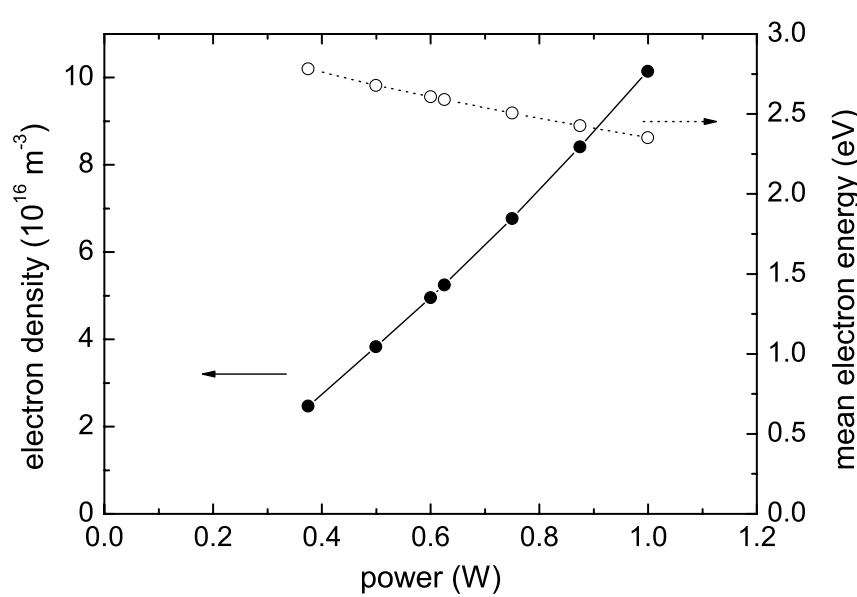

Figure 8. Time and space averaged electron density and mean electron energy for the investigated power range.

for the atomic oxygen density:

$$
n_{\mathrm{O}}=n_{\mathrm{e}} \frac{k_{15}(\epsilon)+2 k_{80}(\epsilon)+k_{81}(\epsilon)}{n_{\mathrm{He}} k_{50}} .
$$

Here, $k_{50}, k_{15}(\epsilon), k_{80}(\epsilon)$ and $k_{81}(\epsilon)$ are the rate coefficients of reactions (R50), (R15), (R80) and (R81), respectively. This electron driven production of ground state atomic oxygen can explain the slightly sub-linear increase in the atomic oxygen density in the numerical simulation (figure 5). The atomic oxygen density depends on the electron density and the mean electron energy, as can be seen in equation (4). Both are shown time and space averaged for the investigated power range in figure 8 . The electron density linearly increases with power, whereas the mean electron energy decreases towards higher powers.

This decrease can be explained by a change in the time and space averaged EEDF [51]. With higher powers, the EEDF at spatio-temporal positions of highest emission (see structures I, II and III in figure 1) is shifted to higher mean electron energies. This leads to a higher number of electrons with sufficient energy to ionize. Hence, the electron density is increased, as illustrated in figure 8. However, the EEDF remains at a low mean electron energy for all other spatiotemporal positions. The time and space averaged EEDF is defined as the sum of the spatio-temporal EEDFs weighted with the corresponding spatio-temporal electron density. The electron density decreases towards the electrode surfaces, hence it stays relatively low at the spatio-temporal positions of highest emission. The percentage of hot electrons in the time and space averaged EEDF therefore decreases with power, resulting in a decreased mean electron energy. The atomic oxygen density is linearly dependent on the electron density but over-linearly dependent on the mean electron energy via the rate coefficients $k_{15}(\epsilon), k_{80}(\epsilon)$ and $k_{81}(\epsilon)$ (see equation (4)). This results in the slightly sub-linear increase in the atomic oxygen density with power, observed in figure 5 .

\section{Conclusions}

A 1D numerical simulation of the $\mu$ APPJ core plasma is presented. In total, 116 reactions among 16 species are accounted for. The model is based on fluid equations with a semi-kinetic treatment of electrons. The kinetic reaction rates and electron transport coefficients are calculated using a two-term approximation Boltzmann solver.

The model predictions for the properties of mid-energy electrons are proven by comparing the simulated spatiotemporal characteristics of the atomic oxygen optical emission line $\lambda=844 \mathrm{~nm}\left({ }^{3} \mathrm{P} \rightarrow{ }^{3} \mathrm{~S}^{\mathrm{o}}\right)$ with corresponding results of experimental PROES measurements. The spatio-temporal structures of both are in very good agreement.

The model predictions for the atomic oxygen density profile across the discharge gap as well as for absolute densities under power variation are compared with TALIF measurements. The simulation supports the experimentally observed flat density profiles and the corresponding conclusion for a volumetric dominated atomic oxygen loss. For the power dependence of absolute densities, both completely independent approaches agree very well.

Finally, a detailed description of the dominant production and destruction mechanisms of the atomic oxygen is given on the basis of the benchmarked numerical simulation. Here, the production is driven by electron-impact reactions and destruction by heavy particle recombination.

\section{Acknowledgments}

The numerical simulations have been supported by Y Sakiyama and DB Graves from the University of California, Berkeley. The authors are grateful to MJ Kushner from the University of Michigan for allocation of electron-impact cross-sections for $\mathrm{O}^{*}$ and $\mathrm{O}_{2}^{*}$. The authors of the Queen's University Belfast acknowledge support from a Science and Innovation Award of the EPSRC (Grant No. EP/D06337X/1) and the authors of the Ruhr-Universität Bochum acknowledge support from the 'Deutsche Forschungsgemeinschaft' with the framework of project A1 in research group FOR1123. One author (LMG) is supported by the DEL.

\section{References}

[1] Baars-Hibbe L, Sichler P, Schrader C, Lucas N, Gericke K H and Büttgenbach S 2005 J. Phys. D: Appl. Phys. 38510

[2] Eden J G et al 2003 J. Phys. D: Appl. Phys. 362869

[3] Aubert X, Bauville G, Guillon J, Lacour B, Puech V and Rousseau A 2007 Plasma Sources Sci. Technol. 1623

[4] Becker K H, Eden J G and Schoenbach K H (ed) 2005 J. Phys. D: Appl. Phys. 38 (cluster issue on microplasmas) http://stacks.iop.org/JphysD/38/i=11

[5] Waskoenig J, O'Connell D, Schulz-von der Gathen V, Winter J, Park S-J and Eden J G 2008 Appl. Phys. Lett. 92101503

[6] Mahony C M O, Gans T, Graham W G, Maguire P D and Petrovic Z Lj 2008 Appl. Phys. Lett. 93011501

[7] Niemi K, Reuter S, Graham L M, Waskoenig J, Knake N, Schulz-von der Gathen V and Gans T 2010 J. Phys. D: Appl. Phys. 43124006

[8] Schaper L, Reuter S, Waskoenig J, Niemi K, Schulz-von der Gathen V and Gans T 2009 J. Phys.: Conf. Ser. 162012013

[9] Schulz-von der Gathen V, Buck V, Gans T, Knake N, Niemi K, Reuter St, Schaper L and Winter J 2007 Contrib. Plasma Phys. 47 510-9 
[10] Stoffels E, Kieft I E, Sladek R E J, van den Bedem L J M, van der Laan E P and Steinbuch M 2006 Plasma Sources Sci. Technol. 15 S169

[11] Foest F, Kindel E, Lange H, Ohl A, Stieber M and Weltmann K D 2007 Contrib. Plasma Phys. 47119

[12] Becker K, Koutsospyros A, Yin S M, Christodoulatos C, Abramzon N, Joaquin J C and Brelles-Marino G 2005 Plasma Phys. Control. Fusion 47 B513

[13] Laroussi M 2009 IEEE Trans. Plasma Sci. 37714

[14] Niemi K, Schulz-von der Gathen V and Döbele H F 2005 Plasma Sources Sci. Technol. 14375

[15] Reuter S, Niemi K, Schulz-von der Gathen V and Döbele H F 2009 Plasma Sources Sci. Technol. 18015006

[16] Knake N, Reuter S, Niemi K, Schulz-von der Gathen V and Winter J 2008 J. Phys. D: Appl. Phys. 41194006

[17] Knake N, Niemi K, Reuter S, Schulz-von der Gathen V and Winter J 2008 Appl. Phys. Lett. 93131503

[18] Niemi K, Reuter S, Schaper L, Knake N, Schulz-von der Gathen V and Gans T 2007 J. Phys.: Conf. Ser. 71012012

[19] Schulz-von der Gathen V, Schaper L, Knake N, Reuter St, Niemi K, Gans T and Winter J 2008 J. Phys. D: Appl. Phys. 41194004

[20] Selwyn G S, Herrmann H W, Park J and Henins I 2001 Contrib. Plasma Phys. 6 610-19

[21] Schütze A, Jeong J Y, Babayan S E, Park J, Selwyn G S and Hicks R F 1998 IEEE Trans. Plasma Sci. 261685

[22] Jeong J Y, Park J, Henins I, Babayan S E, Tu V J, Selwyn G S, Ding G and Hicks R F 2000 J. Phys. Chem. A 1048027

[23] Goehlich A, Kawetzki T and Döbele H F 1998 J. Chem. Phys. 1089362

[24] Gans T, Lin C C, Schulz-von der Gathen V and Döbele H F 2003 Phys. Rev. A 67012707

[25] Abdel-Rahman M, Gans T, Schulz-von der Gathen V and Döbele H F 2005 Plasma Sources Sci. Technol. 1451

[26] Sakiyama Y and Graves D B 2006 J. Phys. D: Appl. Phys. 393644

[27] Stafford D S and Kushner M J 2004 J. Appl. Phys. 962451

[28] Eliasson B and Kogelschatz U 1986 Brown Boveri Technical Report LKR 86-11 C

[29] Yuan X and Raja L L 2003 IEEE Trans. Plasma Sci. 31495

[30] Hagelaar G J M and Pitchford L C 2005 Plasma Sources Sci. Technol. 14722

[31] Vriens L 1964 Phys. Lett. 8260
[32] Bronold F X, Matyash K, Tskhakaya D, Schneider R and Fehske H 2007 J. Phys. D: Appl. Phys. 406583

[33] Phelps A V ftp://jila.colorado.edu/collision data/

[34] Krishnakumar E and Srivastava S K 1992 Int. J. Mass Spectrom. Ion Processes 1131

[35] Matejcik S, Kiendler A, Cicman P, Skalny J, Stampfli P, Illenberger E, Chu Y, Stamatovick A and Märk T D 1997 Plasma Sources Sci. Technol. 6140

[36] Itikawa Y and Ichimura A 1990 J. Phys. Chem. Ref. Data 19637

[37] Lieberman M A and Lichtenberg A J 2005 Principles of Plasma Discharges and Materials Processing 2nd edn (New York: Wiley-Interscience)

[38] Kushner M J private communication

[39] Ellis H W, Pai R Y, McDaniel W, Mason E A and Viehland L A 1976 At. Data Nucl. Data Tables 17177

[40] Sakiyama Y and Graves D B 2007 J. Appl. Phys. 101073306

[41] Shi J J and Kong M G 2005 J. Appl. Phys. 97023306

[42] De Bleecker K, Herrebout D, Bogaerts A, Gijbels R and Descamps P 2003 J. Phys. D: Appl. Phys. 361826

[43] Gomez S, Steen P G and Graham W G 2002 Appl. Phys. Lett. 8119

[44] Waskoenig J, Niemi K, Knake N, Graham L M, Reuter S, Schulz-von der Gathen V and Gans T 2010 Pure Appl. Chem. 82 1209-22

[45] Kee R J, Dixon-Lewis G, Warnatz J, Coltrin M E and Miller J M 1986 Sandia Report SAND86-8246

[46] Shi J J and Kong M G 2005 IEEE Trans. Plasma. Sci. 33624

[47] Waskoenig J and Gans T 2010 Appl. Phys. Lett. 96181501

[48] Comsol multiphysics 3.42007 Herfordshire, AL10 9AB : COMSOL Ltd.

[49] Matlab 7.5 2007 Natick,MA: MathWorks

[50] Gans T, O'Connell D, Schulz-von der Gathen V and Waskoenig J 2010 Plasma Sources Sci. Technol. 19034010

[51] Niemi K, Reuter S, Graham L M, Waskoenig J and Gans T 2009 Appl. Phys. Lett. 95151504

[52] Belenguer Ph and Boeuf J P 1990 Phys. Rev. A 414447

[53] Matyash K, Schneider R, Dittmann K, Meichsner J, Bronold F X and Tskhakaya D 2007 J. Phys. D: Appl. Phys. 406601

[54] Law V J, Daniels S, Walsh J L, Kong M G, Graham L M and Gans T 2010 Plasma Sources Sci. Technol. 19034008 